\title{
ECFMG Certification
}

Last Updated: March 22, 2020

\section{Who Is an International Medical Graduate?}

The Educational Commission for Foreign Medical Graduates (ECFMG) considers anyone who has received his or her basic medical qualification from a recognized university outside the United States or Canada as an international medical graduate (IMG). The citizenship of the physician is not of relevance in this context; a US citizen who has graduated from a foreign university is also considered an IMG, whereas a non-US citizen who graduated from a US or Canadian university is not considered an IMG. Thus, the location of the university is the deciding factor.

\section{Overview-Requirements for ECFMG Certification}

To receive ECFMG certification as an IMG, a person must:

1. Complete the ECFMG application documents

2. Pass Steps 1, 2 CK (clinical knowledge), and 2 CS (clinical skills) of the United States Medical Licensing Examination (USMLE)

3. Obtain verification of medical school credentials, including the following criteria:

a. Minimum of 4 years of medical training at a university 
accredited by the World Directory of Medical Schools

(WDMS)

b. Receipt of your final medical diploma

c. Primary-source verification of your diploma and transcript of records by ECFMG

\section{The USMLE: Steps 1, 2 CK, 2 CS, and 3}

The USMLE is a standardized, uniform test that all medical students in the United States are required to take to determine their eligibility for residency and to eventually practice medicine in the United States. IMGs can apply and take the test through the ECFMG. The test is composed of 3 parts: USMLE Steps 1, 2 CK and 2 CS, and 3.

\section{Step 1}

This is the first and most important part of the USMLE. This part of the test deals with concepts of basic medical science that one usually studies in the first two years of medical school, such as biochemistry, physiology, and anatomy.

Step 1 involves a 1-day computer- and multiple-choicequestion-based test that is divided into 7 blocks. Every block contains around 40 questions that must be answered within 60 minutes, so the test takes a total of nearly 8 hours, including an allowed 45-minute break.

This step is an important part of the residency application. Most residency programs use the score of this test to preselect the most competitive applicants to interview for 
residency positions.

The current USMLE Step 1 test fee is $\$ 940$ per exam registration.

\section{Step 2}

The second part of the USMLE is composed of 2 tests, the Step 2 CK and Step 2 CS.

Step 2 CK (clinical knowledge) assesses knowledge on clinical medical specialties such as internal medicine, pediatrics, surgery, and radiology. It assesses how well one can apply his or her clinical knowledge in clinical practice. This 1-day test is 1 hour longer than that in Step 1; it takes 9 hours to complete and is divided into 8 60-minute blocks.

The current USMLE Step 2 CK test fee is $\$ 940$ per each exam registration.

Step 2 CS (clinical skills) tests one's ability to apply the theoretical clinical concepts that he or she has acquired during medical school in a clinical setting, including physical examination, history taking, and bedside communication skills. The test taker interacts with 12 experienced actors whose job is to act like a patient, and these actors will then evaluate performance on the basis of the test taker's interactions with them. Part of this test is also computer based, in that the test taker must type clinical notes and a treatment plan for each case into a computer. 
The current USMLE Step 2 CS test fee is $\$ 1,580$ per exam registration.

\section{Step 3}

Step 3 is the final part of the USMLE series. Whereas Steps 1 and 2 test the ability to practice medicine in a supervised setting, Step 3 tests how well one can apply basic medical and clinical knowledge in an unsupervised setting.

This is a 2-day test: on day 1 is Foundations of Independent Practice (FIP), which includes 232 multiple-choice questions in 6 blocks of 60 minutes each, and on day 2 is Advanced Clinical Medicine (ACM), which includes 180 multiple-choice questions in 6 blocks of 60 minutes each.

The current USMLE Step 3 test fee is $\$ 850$ per exam registration.

\section{Preparing for Exams}

To prepare for the exams, one first must decide on the resource material. Different books and question banks are available for each of the exams. The following resources are suggested on the basis of previous applicants' experiences. One may also choose to use additional or other helpful resources and study material.

\section{Preparing for Step 1}

The most important and valuable resources are First Aid for the USMLE Step 1 and the online question bank of UWorld. As 
you prepare, you can add resources such as online tutoring (eg, Pathoma) and other online question banks.

Everyone has a different style of studying and preparing for the exam, but on the basis of previous applicants' experience, the First Aid and UWorld materials are a must. You can make a schedule for how you want to cover the topics and practice on UWorld. A general recommendation is to read First Aid; then, read it again, and start working with UWorld, covering 1 block of 40 questions each per day, and re-review each block.

Once you are done with UWorld, you can either review it again or cover the questions again, depending on what you think will help your preparation most. UWorld allows you to flag what you think are important questions for later review, and it allows you to review your wrong answers.

After finishing the UWorld questions, you can do practice tests that will return a predicted score. These scores might be underpredictive or overpredictive, but they will provide a good, generalized idea of where your preparation stands. When you get a score above the one you desire in one of these practice tests, that is usually a good time to take the actual exam. Some practice tests are available on the UWorld website, and others are available on the National Board of Medical Examiners (NBME) website. These are the 2 most common practice test resources used by medical students.

\section{Preparing for Step 2 CK}

The preparation for this exam is similar to that for Step 1. 
One option is to use a book such as First Aid for the USMLE Step 2 CK, Master the Boards Step 2 CK, or any of the others available. The most important resource for the Step $2 \mathrm{CK}$ is UWorld. You should aim to understand UWorld well and, ideally, go through it at least twice.

You can also use other available online question banks, such as Rx and Kaplan. After reviewing these resources, you can take practice tests similar to the ones for Step 1, which also will provide a predicted score. On the basis of this score, you can analyze your performance and determine a good time to take the test.

\section{Preparing for Step 2 CS}

It would be helpful to have at least one study partner, ideally someone who is also studying for the exam. First Aid for the USMLE Step 2 CS is a great book to use. It has practice cases that you can review with your study partner. For each practice case, one of you will be the physician, and the other will be the patient. First Aid has extensive information for IMGs on how to prepare for the exam, so read it thoroughly, and practice it well.

\section{USMLE Performance Required for Neurosurgery Residency}

The Step 1 score is one of the most important factors that can increase your chances of getting accepted to a US program. The higher the score, the better. Most of the program directors use it as a screening and selection tool for residency interviews. 
The mean USMLE scores in 2018 reported by the National Resident Matching Program (NRMP) for those who were accepted into a neurosurgery residency program were 246 for Step 1 and 243 for Step 2. For IMGs, a higher score is encouraged, and a Step 1 score of higher than 260 will greatly increase the chances of being matched into neurosurgery; on the basis of recent NRMP data, one's chances are around $45 \%$ with this score.

\section{ECFMG Certification Application}

The certification process starts with an application for a USMLE/ECFMG identification number through the ECFMG interactive web application. As soon as you receive this number, you should include it on all forms, payments, and documents. The application cannot be completed offline.

For certification, you must provide your name, date of birth, and sex. Medical students are required to confirm their enrollment in a foreign university included in the WDMS. The prospective graduation year must also be completed. You will have to release certain legal claims and confirm your understanding of the purpose of the ECFMG certification.

As soon as the required information is supplied, it usually becomes part of an IMGs permanent ECFMG record, and the application becomes valid immediately. This will allow you to apply for the USMLE. If you submitted an ECFMG certification application as a medical student, there is no need to submit another application after medical school graduation. 
The standard ECFMG certificate will include your name, certificate number, examination dates, and the date on which the ECFMG certification was issued. The current application fee is $\$ 135$.

\section{Additional Online Resources}

- ECFMG certification verification service: http://www.ecfmg.org/cvs

- ECFMG certification factsheet: https://www.ecfmg.org/forms/certfact.pdf

- ECFMG and USMLE application portal: https://secure2.ecfmg.org/emain.asp?app=iwa

- Overview of fees: https://www.ecfmg.org/fees/index.html

- WDMS: https://www.wdoms.org/

\section{Contributors}

Ahmed Habib, MD

Aida Kafai Golahmadi, BS

Ayesha Quddusi, MD

Saad R. Alghamdi, MD

Victor E. Staartjes, BMed

Vittorio Stumpo, MD 


\section{DOI: https://www.doi.org/10.18791/nsatlas.v13.2}

\section{References}

1. National Resident Matching Program. Charting outcomes in the match: international medical graduates. Available at: http://www.nrmp.org/wp-

content/uploads/2018/06/Charting-Outcomes-in-theMatch-2018-IMGs.pdf. Accessed March 16, 2019.

\section{Submit Your \\ Complex Case \\ to be reviewed by the Atlas team.}




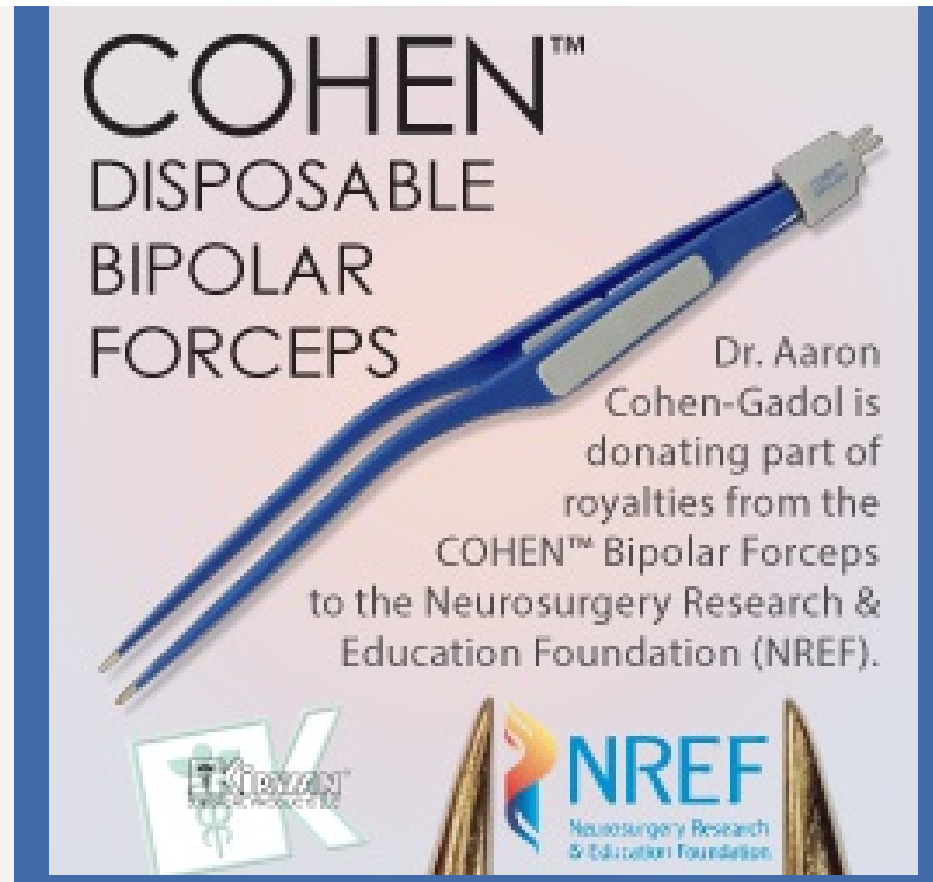

